\title{
Variação sazonal do gaivotão (Larus dominicanus) durante o ano de 2005 no estuário de Cananéia-Iguape-Ilha Comprida, São Paulo, Brasil
}

\author{
Edison Barbieri ${ }^{1,2}$ \\ ${ }^{1}$ Instituto de Pesca da Secretaria da Agricultura e Abastecimento do Estado de São Paulo, \\ CP 61, CEP 11990-000, Cananéia, SP, Brasil \\ ${ }^{2}$ Autor para correspondência: Edison Barbieri,e-mail: edisonbarbieri@yahoo.com.br
}

BARBIERI, E. 2008. The gull (Larus dominicanus) distribution during the year of the 2005 at CananéiaIguape-Ilha Comprida estuary, São Paulo, Brazil. Biota Neotrop. 8(2): http://www.biotaneotropica.org.br/ v8n2/en/abstract?article+bn01708022008.

Abstract: This work analyzed Larus dominicanus's abundance and its seasonal variation along 2005 at CananéiaIguape-Ilha Comprida estuary, southern São Paulo, Brazil. The gulls were found throughout the year at the study area. Censuses were made from January to December 2005, on a weekly basis. A total of 48 census were realized in each five areas of the estuary. The results show the L. dominicanus is a common species at the estuary and the number of individuals varied among the sites. The lowest numbers were found in the winter, agreeing with the nesting season of the local population. During late summer and early spring the numbers increased.

Keywords: seasonal variation, abundance, estuary, Larus dominicanus, seabird.

BARBIERI, E. 2008. Variação sazonal do gaivotão (Larus dominicanus) durante o ano de 2005 no estuário de Cananéia-Iguape-Ilha Comprida, São Paulo. Biota Neotrop. 8(2): http://www.biotaneotropica.org.br/v8n2/ pt/abstract?article+bn01708022008.

Resumo: Este trabalho analisou a abundância e variação sazonal de Larus dominicanus durante o ano de 2005 no estuário de Cananéia-Iguape-Ilha Comprida, litoral sul do estado de São Paulo. O gaivotão foi uma ave constante durante todo o ano no estuário. As contagens numéricas foram realizadas de janeiro a dezembro de $2005 \mathrm{em}$ visitas semanais, totalizando 48 censos em cada uma das cinco áreas escolhidas para este estudo. Os resultados indicaram que o gaivotão foi uma espécie freqüente no estuário e que o número de indivíduos variou em função da área estudada. No geral, a espécie foi abundante no verão, ocorrendo uma diminuição do número de indivíduos durante o final do outono e início do inverno.

Palavras-Chave: variação sazonal, abundância, Larus dominicanus, estuário, aves marinhas. 


\section{Introdução}

O gaivotão (Larus dominicanus, Charadriiformes: Laridae), habita o litoral e ilhas costeiras do Pacífico e Atlântico sul-americano; da Terra do Fogo até norte do Peru, na costa do Brasil são encontrados do Rio Grande do Sul até o Espírito Santo (Sick 1997, Novelli 1997). Também é encontrado no Sul da África, Austrália, Nova Zelândia e em ilhas Subantárticas (Watson 1975). Quanto ao habito alimentar a espécie é descrita como, predadora, necrófaga e cleptoparasita intra e interespecífico (Hockey et al. 1989, Sick 1997), com uma marcada tendência ao oportunismo alimentar (Favero et al. 1997). O comportamento alimentar de L. dominicanus está estreitamente ligado à presença de pequenos peixes próximos às suas áreas de nidificação (Burger \& Gochfeld 1996, Sick 1997, Novelli 1997). O gaivotão obtém seus recursos alimentares na zona de arrebentação e nas praias (supra e mesolitoral), onde são comumente vistos em terra se alimentando de restos de animais mortos e de lixo deixado pelo homem (Sick 1997). O aumento da população do gaivotão, em alguns casos, foi atribuído à disponibilidade de alimento encontrado em lixo de origem humana (Crawford et al. 1982). Mamíferos marinhos mortos encontrados na praia são também fontes de alimentos para essas aves (Novelli 1997).

Larus dominicanus, podem ser vistos em pequenos e grandes bandos sobre as rochas costeiras no Rio de Janeiro (Sick 1997) e repousando na praia da Ilha Comprida, no litoral sul de São Paulo (Barbieri \& Mendonça 2008). Em Santa Catarina e Rio Grande do Sul é observada durante todo o ano descansando e se alimentando nas praias (Vooren \& Brusque 1999, Branco \& Eliot 2002, Rosário 2004). Essa espécie é comum no estuário de Cananéia-Iguape-Ilha Comprida, região com alta produtividade primária, e conseqüentemente, muito abundante em invertebrados, peixes, aves de praia e marinhas (Barbieri et al. 2003, Barbieri \& Pinna 2005).

Este trabalho teve por objetivo analisar a variação mensal, sazonal e a relação com a pesca de camarão-sete-barbas (Xiphopenaeus kroyeri) e a abundância de Larus dominicanus, em cinco áreas do estuário de Cananéia-Iguape-Ilha Comprida, durante o ano de 2005.

\section{Material e Métodos}

O Trabalho foi realizado em cinco áreas do Complexo Estuarino Lagunar Cananéia-Iguape-Ilha Comprida, litoral sul do Estado de São Paulo (Figura 1). Para a escolha dos locais de amostragens foram

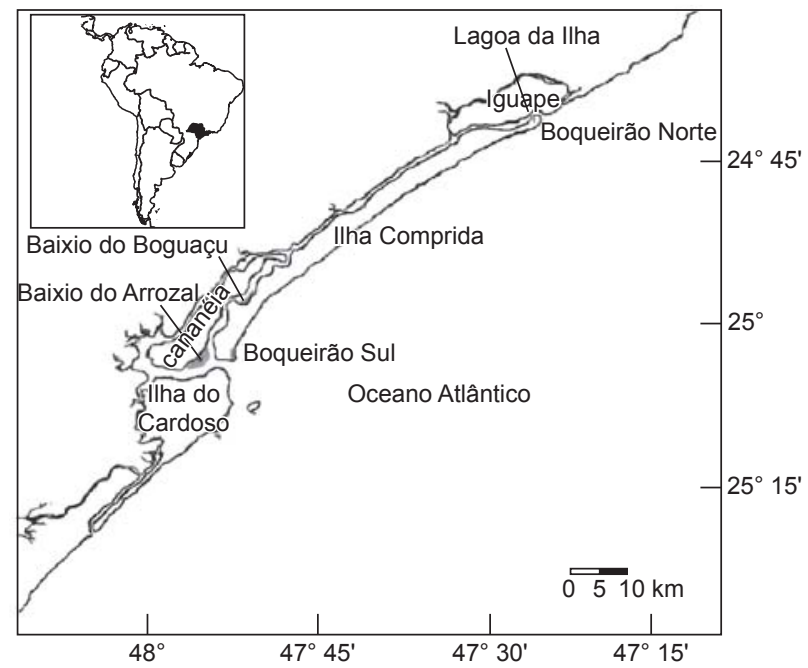

Figura 1. Localização do estuário de Cananéia-Iguape-Ilha Comprida com as áreas de estudo no litoral sul do Estado de São Paulo.

Figure 1. Cananéia-Iguape-Ilha Comprida estuary with the areas of study in the south coast of the State of São Paulo. considerados: a abundância dos bandos de Larus dominicanus, a localização geográfica e a possibilidade de acesso ao longo do ano, sendo selecionadas cinco localidades: Ponta da Praia do Boqueirão Sul $\left(25,05455^{\circ} \mathrm{S}\right.$ e $\left.47,94221^{\circ} \mathrm{W}\right)$, Ponta da praia do Boqueirão Norte $\left(24,68409^{\circ} \mathrm{S}\right.$ e $\left.47,4272^{\circ} \mathrm{W}\right)$, Lagoa da Ilha $\left(24,68155^{\circ} \mathrm{S}\right.$ e $\left.47,43909^{\circ} \mathrm{W}\right)$, Baixio do Arrozal $\left(25,05455^{\circ} \mathrm{S}\right.$ e $\left.47,94221^{\circ} \mathrm{W}\right) \mathrm{e}$ Baixio do Boguaçu $\left(24,96865^{\circ} \mathrm{S}\right.$ e $\left.47,89753^{\circ} \mathrm{W}\right)$.

A pesquisa teve duração de 12 meses de coleta, sendo realizada entre janeiro a dezembro de 2005, com censos semanais, totalizando 48 amostragens em cada uma das áreas escolhidas. Os Censos foram realizados nas praias e nos bancos de areia dos baixios, durante maré baixa através de contagem direta, segundo a metodologia descrita por Bibby et al. (1992), onde o observador em um ponto fixo desenvolvia contagem individual de cada espécie com auxílio de luneta Bouch \& Lomb $(20 \times 60)$, binóculos $(10 \times 50)$ e $(15 \times 90 \times 180)$. Na Ponta da Praia do Boqueirão Sul e na Ponta da praia do Boqueirão Norte, juntamente com os censos foram, contados os barcos envolvidos na pesca do camarão-sete-barbas que estavam atuando próximos à praia (sendo facilmente avistados). Após esse procedimento, fez-se a correlação entre o número de barcos camaroneiros com o número de indivíduos de L. dominicanus. Considerou-se Forte Correlação para os valores obtidos entre 0,70 e 0,89 (Fowler \& Cohen 1988). Também, fez-se a variação da produção do camarão-sete-barbas (Xiphopenaeus kroyeri) e das médias de indivíduos de L. dominicanus, para identificar qual era a tendência. A Constância foi calculada com base na fórmula: $\mathrm{C}=\mathrm{p} \times 100 / \mathrm{P}$ onde $\mathrm{p}$ corresponde ao número de visitas que a espécie foi avistada e $P$ refere-se ao número total de visitas. A flutuação sazonal foi avaliada pelas médias das contagens mensais. Em todo o estuário as abundâncias médias mensal e sazonal de $L$. dominicanus, foram examinadas através de um teste de variância (ANOVA, $p>0,05$ ).

\section{Resultados}

O gaivotão foi uma espécie freqüente no estuário de CananéiaIguape e Ilha Comprida, sendo registrado em todos os meses de 2005. No Boqueirão Sul o maior número de indivíduos foi observado em Janeiro, Fevereiro e Dezembro com 3142; 5041 e 3027 aves respectivamente (Tabela 1). Observa-se, entretanto, um declínio da população a partir de Março, que permaneceu baixa até Outubro (Figura 2).

Os censos realizados no Baixio do Arrozal mostraram um aumento gradual da espécie a partir do mês de Novembro; quando foram avistados 68 indivíduos (Figura 3). Houve, no entanto, uma queda dessa população a partir de Março $(13,66)$, que se manteve baixa até Outubro (Figura 3). A menor média registrada foi em Setembro, com 4 indivíduos (Tabela 2).

$\mathrm{Na}$ Lagoa da Ilha observou-se um maior número de gaivotões nos meses de Novembro, com 35 indivíduos e Dezembro, com 33 (Tabela 3). A população começou a diminuir a partir de Abril e manteve-se baixa durante o inverno, sendo que no mês de Junho nenhum espécime foi observado (Figura 4).

No Boqueirão Norte observou-se baixas médias entre os meses de Março a Outubro. Em Março não foi registrado nenhum indivíduo e em Junho apenas um. A população de Larus dominicanus nesta área seguiu o mesmo padrão das observadas para o Boqueirão Sul, embora com médias bem menores (Tabela 4). Houve um aumento nos meses mais quentes Janeiro e Dezembro (Figura 5), com registros de 60 e 74 indivíduos.

Para o Baixio do Boguaçu os maiores registros da população ocorreram nos meses de Abril e Agosto com 49 e 77 indivíduos respectivamente (Tabela 5); e assim como no Arrozal, observou-se uma queda do número de aves a partir de Maio, mantendo-se baixa até Junho (Figura 6).

A análise da Figura 7 mostra uma queda brusca do número de aves a partir do mês de Março, para as 5 áreas combinadas. A população do gaivotão foi maior durante o verão em relação às demais estações do ano. 
Tabela 1. Número total de indivíduos de Larus dominicanus avistados em 2005 no Boqueirão Sul, com as respectivas médias, desvio padrão e frequiência de ocorrência $(\%)$.

Table 1. Total number of Larus dominicanus sighted in 2005 in the Boqueirão Sul, with the respective averages, standard deviations and frequency of occurrence $(\%)$.

\begin{tabular}{lcccc}
\hline \multicolumn{1}{c}{ Meses } & $\begin{array}{c}\mathbf{N}^{\mathbf{0}} \text { total de } \\
\text { indivíduos }\end{array}$ & Médias & $\begin{array}{c}\text { Desvio } \\
\text { Padrão }( \pm)\end{array}$ & Constancia (\%) \\
\hline Janeiro & 3142 & 584,91 & 62,88 & 100 \\
Fevereiro & 5041 & 1003,5 & 219,62 & 100 \\
Março & 1399 & 236,08 & 60,31 & 100 \\
Abril & 238 & 119,08 & 48,67 & 100 \\
Maio & 283 & 59,78 & 10,22 & 100 \\
Junho & 374 & 176,25 & 90,64 & 100 \\
Julho & 159 & 82,36 & 27,57 & 100 \\
Agosto & 166 & 49,30 & 13,60 & 100 \\
Setembro & 252 & 82,58 & 17,73 & 100 \\
Outubro & 251 & 175,80 & 30,43 & 100 \\
Novembro & 2612 & 479,61 & 76,21 & 100 \\
Dezembro & 3027 & 829,54 & 120,41 & 100 \\
\hline
\end{tabular}

Tabela 2. Número total de indivíduos de Larus dominicanus avistados em 2005 no Baixio do Arrozal, com as respectivas médias, desvio padrão e frequiência de ocorrência (\%).

Table 2. Total number of Larus dominicanus sighted in 2005 in the Baixio do Arrozal, with the respective averages, standard deviations and frequency of occurrence $(\%)$.

\begin{tabular}{lcccc}
\hline Meses & $\begin{array}{c}\mathbf{N}^{\circ} \text { total de } \\
\text { indivíduos }\end{array}$ & Médias & $\begin{array}{c}\text { Desvio } \\
\text { Padrão }( \pm)\end{array}$ & Constancia (\%) \\
\hline Janeiro & 176 & 58,66 & 16,22 & 100 \\
Fevereiro & 81 & 27,00 & 17,24 & 100 \\
Março & 82 & 13,66 & 3,82 & 100 \\
Abril & 28 & 14,00 & 9,00 & 100 \\
Maio & 30 & 7,50 & 2,90 & 100 \\
Junho & 48 & 9,60 & 3,35 & 100 \\
Julho & 17 & 8,50 & 2,50 & 100 \\
Agosto & 26 & 8,66 & 4,97 & 100 \\
Setembro & 12 & 4,00 & 0,57 & 100 \\
Outubro & 25 & 5,00 & 1,51 & 100 \\
Novembro & 68 & 13,60 & 4,77 & 100 \\
Dezembro & 115 & 23,00 & 6,94 & 100 \\
\hline
\end{tabular}

Tabela 3. Número total de indivíduos de Larus dominicanus avistados em 2005 na Lagoa da Ilha, com as respectivas médias, desvio padrão e frequiência de ocorrência $(\%)$.

Table 3. Total number of Larus dominicanus sighted in 2005 in the Lagoa da Ilha, with the respective averages, standard deviations and frequency of occurrence $(\%)$.

\begin{tabular}{lcccc}
\hline \multicolumn{1}{c}{ Meses } & $\begin{array}{c}\mathbf{N}^{\mathbf{}} \text { total de } \\
\text { indivíduos }\end{array}$ & Médias & $\begin{array}{c}\text { Desvio } \\
\text { Padrão }( \pm)\end{array}$ & $\begin{array}{c}\text { Freqüência de } \\
\text { ocorrência (\%) }\end{array}$ \\
\hline Janeiro & 18 & 6,00 & 2,08 & 75 \\
Fevereiro & 11 & 2,75 & 2,42 & 25 \\
Março & 9 & 2,25 & 1,31 & 25 \\
Abril & 17 & 5,66 & 2,33 & 25 \\
Maio & 3 & 1,00 & 1,00 & 25 \\
Junho & 0 & 0,00 & 0,00 & 0 \\
Julho & 4 & 1,33 & 0,66 & 25 \\
Agosto & 2 & 0,66 & 0,66 & 25 \\
Setembro & 16 & 5,33 & 3,17 & 50 \\
Outubro & 13 & 4,33 & 1,20 & 50 \\
Novembro & 35 & 11,66 & 1,85 & 100 \\
Dezembro & 33 & 11,00 & 2,08 & 100 \\
\hline
\end{tabular}

Tabela 4. Número total de indivíduos de Larus dominicanus avistados em 2005 no Boqueirão Norte, com as respectivas médias, desvio padrão e freqüência de ocorrência $(\%)$.

Table 4. Total number of Larus dominicanus sighted in 2005 in the Boqueirão Norte, with the respective averages, standard deviations and frequency of occurrence $(\%)$.

\begin{tabular}{lcrcc}
\hline Meses & $\begin{array}{c}\mathbf{N}^{\mathbf{0}} \text { total de } \\
\text { indivíduos }\end{array}$ & Médias & $\begin{array}{c}\text { Desvio } \\
\text { Padrão }( \pm)\end{array}$ & $\begin{array}{c}\text { Freqüência de } \\
\text { ocorrência }(\%)\end{array}$ \\
\hline Janeiro & 60 & 15,00 & 6,17 & 100 \\
Fevereiro & 23 & 5,75 & 2,95 & 75 \\
Março & 12 & 3,00 & 1,22 & 25 \\
Abril & 14 & 3,50 & 1,75 & 25 \\
Maio & 0 & 0,00 & 0,00 & 0 \\
Junho & 1 & 0,25 & 0,25 & 25 \\
Julho & 25 & 6,25 & 1,75 & 25 \\
Agosto & 8 & 2,00 & 0,70 & 25 \\
Setembro & 11 & 2,75 & 0,62 & 50 \\
Outubro & 2 & 0,50 & 0,50 & 25 \\
Novembro & 28 & 7,00 & 1,22 & 75 \\
Dezembro & 74 & 18,50 & 3,84 & 75 \\
\hline
\end{tabular}

Tabela 5. Número total de indivíduos de Larus dominicanus avistados em 2005 no Baxio do Boguaçu, com as respectivas médias, desvio padrão e frequiência de ocorrência (\%).

Table 5. Total number of Larus dominicanus sighted in 2005 in the Baxio do Boguaçu, with the respective averages, standard deviations and frequency of occurrence $(\%)$.

\begin{tabular}{lcccc}
\hline Meses & $\begin{array}{c}\mathbf{N}^{\mathbf{0}} \text { total de } \\
\text { indivíduos }\end{array}$ & Médias & $\begin{array}{c}\text { Desvio } \\
\text { Padrão }( \pm)\end{array}$ & $\begin{array}{c}\text { Freqüência de } \\
\text { ocorrência }(\%)\end{array}$ \\
\hline Janeiro & 32 & 6,40 & 3,07 & 75 \\
Fevereiro & 16 & 4,00 & 2,73 & 25 \\
Março & 28 & 4,66 & 1,25 & 25 \\
Abril & 49 & 8,16 & 2,50 & 100 \\
Maio & 25 & 3,57 & 1,37 & 50 \\
Junho & 12 & 2,00 & 1,29 & 25 \\
Julho & 11 & 1,83 & 0,79 & 25 \\
Agosto & 77 & 15,40 & 5,40 & 100 \\
Setembro & 14 & 2,80 & 1,24 & 50 \\
Outubro & 5 & 1,25 & 1,25 & 25 \\
Novembro & 13 & 3,25 & 1,88 & 25 \\
Dezembro & 24 & 6,00 & 2,58 & 50 \\
\hline
\end{tabular}

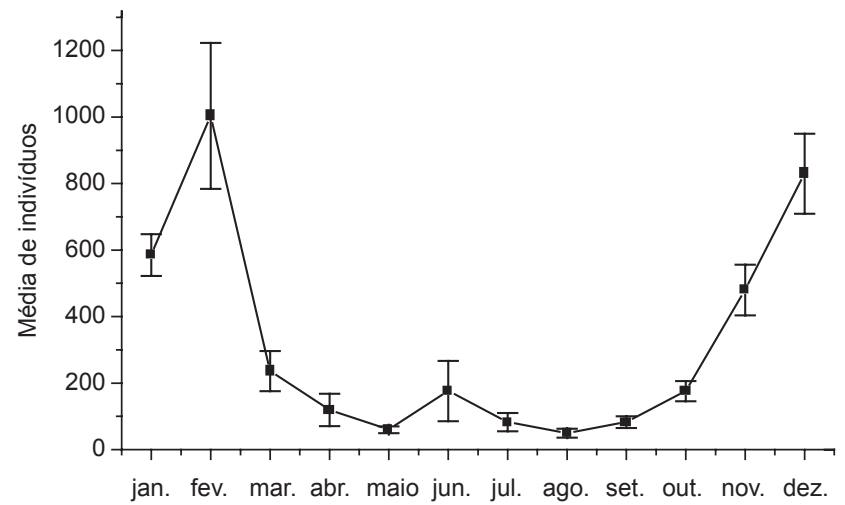

Figura 2. Variação mensal de indivíduos de Larus dominicanus no baixio no Boqueirão Sul em 2005. Os valores correspondem à média de 4 amostras feitas mensalmente. As barras são os respectivos desvios padrões.

Figure 2. Individual averages of Larus dominicanus in the Baixio do Boqueirão Sul. The values correspond to the averages of 4 samplings per month. The bars are the respective standard deviations. 


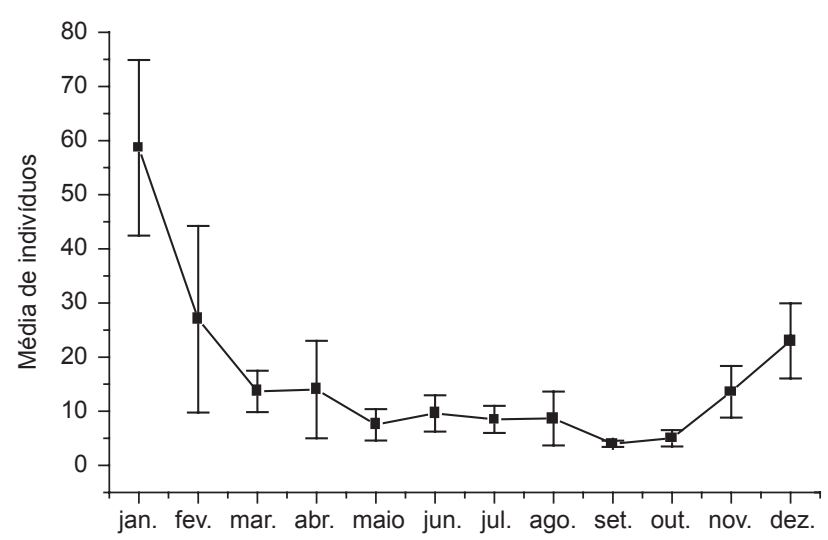

Figura 3. Variação mensal de indivíduos de Larus dominicanus no baixio do Arrozal em 2005. Os valores correspondem à média de 4 amostras feitas mensalmente. As barras são os respectivos desvios padrões

Figure 3. Individual averages of Larus dominicanus in the Baixio do Arrozal. The values correspond to the averages of 4 samplings per month. The bars are the respective standard deviations.

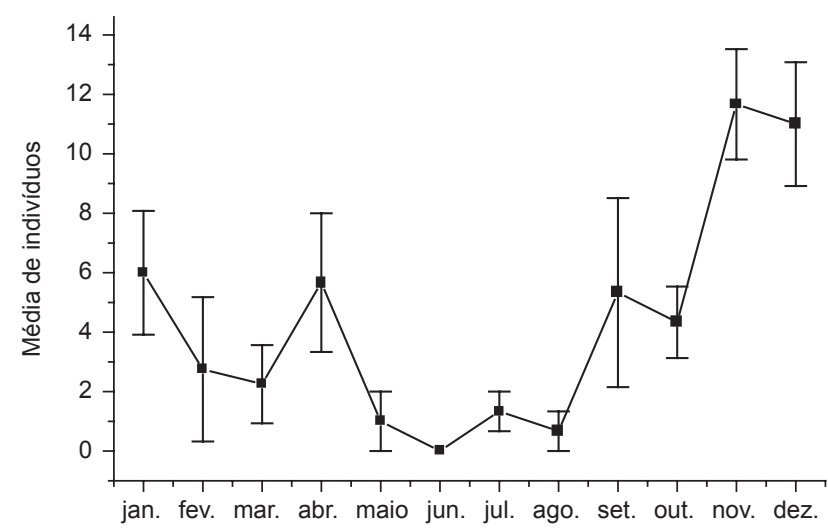

Figura 4. Variação mensal de indivíduos de Larus dominicanus na Lagoa da Ilha em 2005. Os valores correspondem à média de 4 amostras feitas mensalmente. As barras são os respectivos desvios padrões.

Figure 4. Individual averages of Larus dominicanus in the Lagoa da Ilha. The values correspond to the averages of 4 samplings per month. The bars are the respective standard deviations.

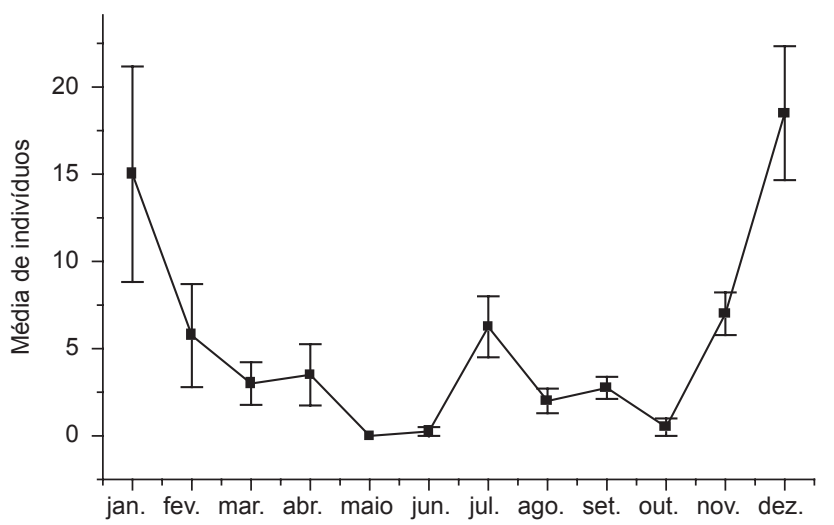

Figura 5. Variação mensal de indivíduos de Larus dominicanus no Boqueirão Norte em 2005. Os valores correspondem à média de 4 amostras feitas mensalmente. As barras são os respectivos desvios padrões.

Figure 5. Individual averages of Larus dominicanus in the Boqueirão Norte. The values correspond to the averages of 4 samplings per month. The bars are the respective standard deviations.

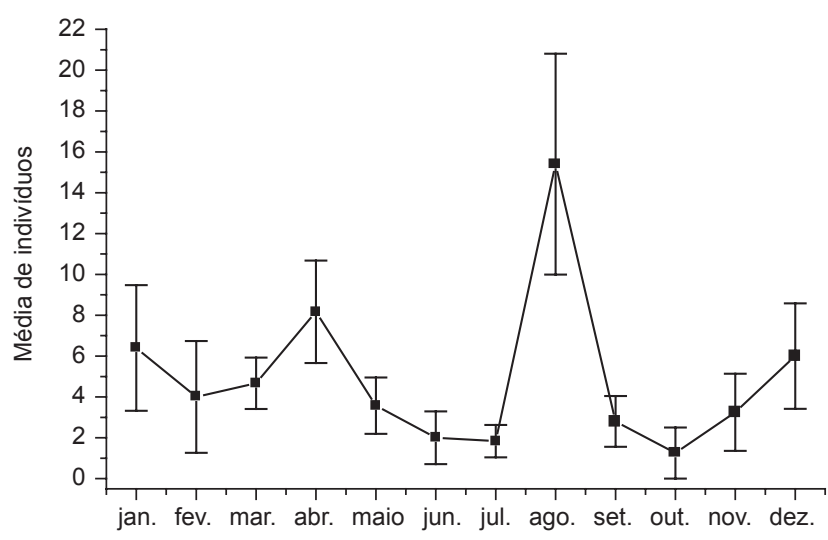

Figura 6. Variação mensal de indivíduos de Larus dominicanus no baixio do Baixio do Boguaçu em 2005. Os valores correspondem à média de 4 amostras feitas mensalmente. As barras são os respectivos desvios padrões.

Figure 6. Individual averages of Larus dominicanus in the Baixio do Boguaçu. The values correspond to the averages of 4 samplings per month. The bars are the respective standard deviations.

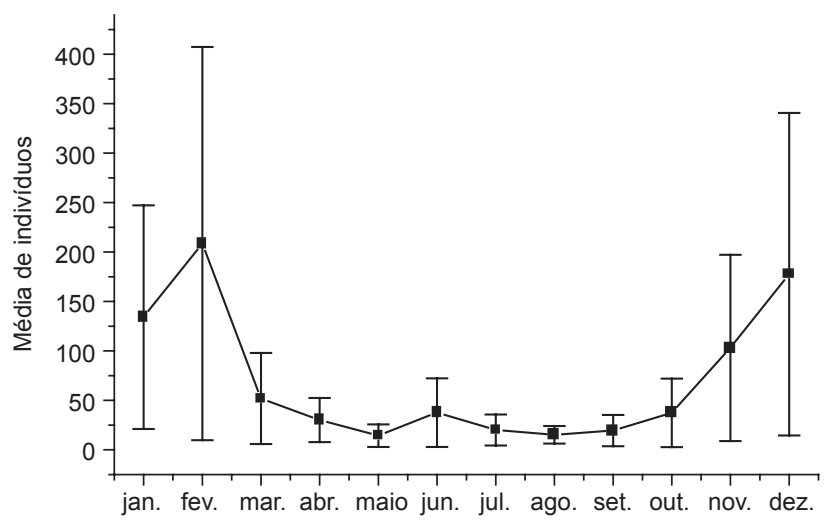

Figura 7. Variação mensal de indivíduos de Larus dominicanus nas cinco áreas combinadas do Estuário de Cananéia-Iguape-Ilha Comprida em 2005. Os valores correspondem à média de 4 amostras feitas mensalmente. As barras são os respectivos desvios padrões.

Figure 7. Individual averages of Larus dominicanus in the five agreed areas of the Cananéia-Iguape-Ilha Comprida estuary. The bars are the respective standard deviations.

Entretanto, o mesmo não ocorreu no Baixio do Boguaçu, onde foi visto um maior número da espécie durante o Inverno (Figura 7). Observou-se que, para os cinco pontos amostrados, após ocorrer uma queda brusca da população no mês Março, esta se manteve baixa até Outubro.

Em relação a sazonalidade, foi possível registrar um aumento da população nas estações de verão e primavera e uma pronunciada diminuição no outono e inverno (Figura 8). Comparando-se as médias sazonais, não houve diferença significativa $(\mathrm{p}>0,05)$ entre o verão e primavera e entre outono e o inverno. Verão e primavera foram, entretanto, estatisticamente diferentes do outono e do inverno.

Foi possível identificar dois locais principais de concentração do gaivotão na região estuarina trabalhada. A localidade do Boqueirão Sul possuiu meses com médias superiores a 200 indivíduos presentes e a área do Baixio do Arrozal onde a Constância foi de $100 \%$ em todos os meses estudados. Os outros pontos apresentaram médias mensais abaixo de 30 indivíduos da espécie. O terceiro ponto de censo na região meridional, Boguaçu, cerca de $10 \mathrm{~km}$ de distância dos outros dois, virtualmente foi inexpressivo regionalmente como local de pouso para a espécie, com médias mensais de dois gaivotões ou ainda menores. Combinando-se os dados de todos os pontos trabalhados em 


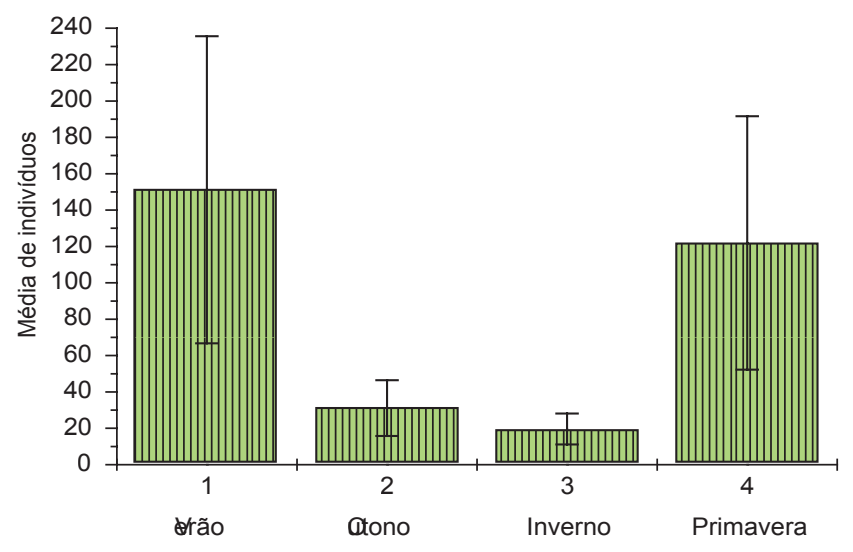

Figura 8. Variação sazonal de indivíduos de Larus dominicanus nas cinco áreas combinadas do Estuário de Cananéia-Iguape-Ilha Comprida em 2005. As barras são os respectivos desvios padrões.

Figure 8. Seasonal variation of Larus dominicanus in the five agreed areas of the Cananéia-Iguape-Ilha Comprida estuary. The bars are the respective standard deviations.

uma única média mensal para a região da Ilha Comprida (Figura 7), nota-se uma sobreposição do desvio padrão mensal, e uma diminuição no número de indivíduos de Março a Outubro.

A correlação entre o número de barcos camaroneiros envolvidos na pesca do camarão-sete-barbas (Xiphopenaeus kroyeri) e o número de indivíduos de Larus dominicanus foi forte $\left(\mathrm{r}=0,81, \mathrm{p}=5,91.10^{-10}\right.$, Figura 9). A variação da produção do camarão-sete-barbas e o número de indivíduos de $L$. dominicanus nas cinco áreas combinadas registraram uma tendência das aves acompanharem a abundância do recurso pesqueiro (Figura 10).

Além da interação com os barcos de pesca, indivíduos de Larus dominicanus também foram avistados interagindo com o Boto-cinza (Sotalia fluviatilis guianensis), quando pescavam na baía de Trapandé e nas proximidades do baixio do Boguaçu.

\section{Discussão}

As gaivotas são um importante componente da comunidade de aves marinhas que se alimentam nas regiões intermareais (Moreira 1995, Burger \& Gochfeld 1996). No estuário de Cananéia-Iguape-Ilha Comprida, Larus dominicanus é uma das espécies de aves marinhas mais abundante e de ampla distribuição (Barbieri \& Mendonça 2008). São abundantes principalmente na primavera e no verão como foi evidenciado neste trabalho.

Larus dominicanus reproduz no inverno em ilhas costeiras da Região Tropical Sul do Brasil, e populações austrais destas espécies nidificam na primavera nas costas do Uruguai e Argentina (Escalante 1970). Aves destas populações ocorrem como migrantes de inverno nas Regiões Subtropical e Tropical Sul do Brasil (Vooren \& Brusque 1999). As flutuações sazonais na abundância da espécie no estuário de Cananéia-Iguape-Ilha Comprida estão, provavelmente, relacionadas com o período reprodutivo. Segundo Branco e Ebert (2002) as menores abundâncias registradas no estuário do Saco da Fazenda (Santa Catarina) foram entre julho a outubro, refletindo o deslocamento dos adultos para as áreas de reprodução; enquanto que a tendência de incremento entre outubro até janeiro indicou o retorno dos adultos e o ingresso de indivíduos jovens na área. Essa suposição é reforçada por Giaccardi et al. (1997), que estudou a população de L. dominicanus nos depósitos de lixo de Rawson, Argentina, verificando que as menores abundâncias na área coincidem com a temporada reprodutiva da espécie na Patagônia.

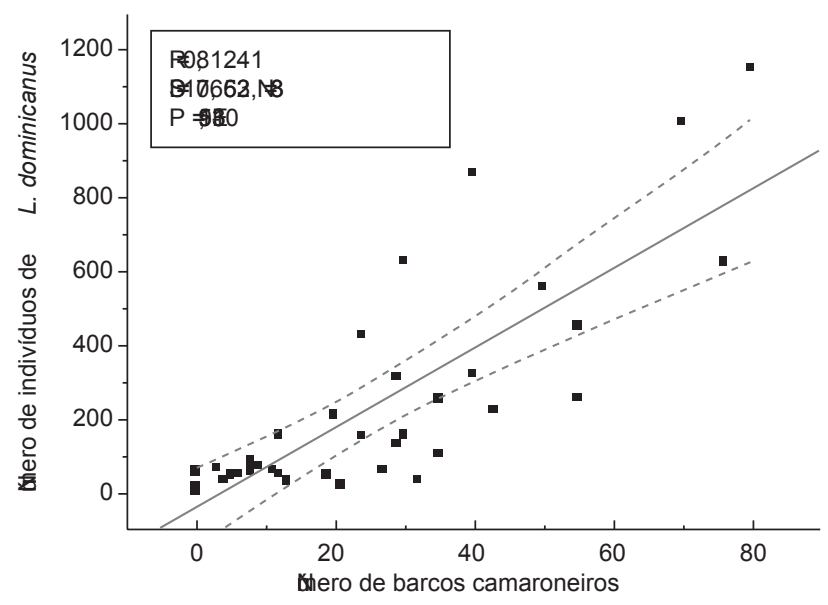

Figura 9. Correlação entre o número de barcos camaroneiros e o número de indivíduos de Larus dominicanus no Boqueirão Sul.

Figure 9. Correlation between the number of fishing boats and individual number of Larus dominicanus in the Boqueirão Sul.
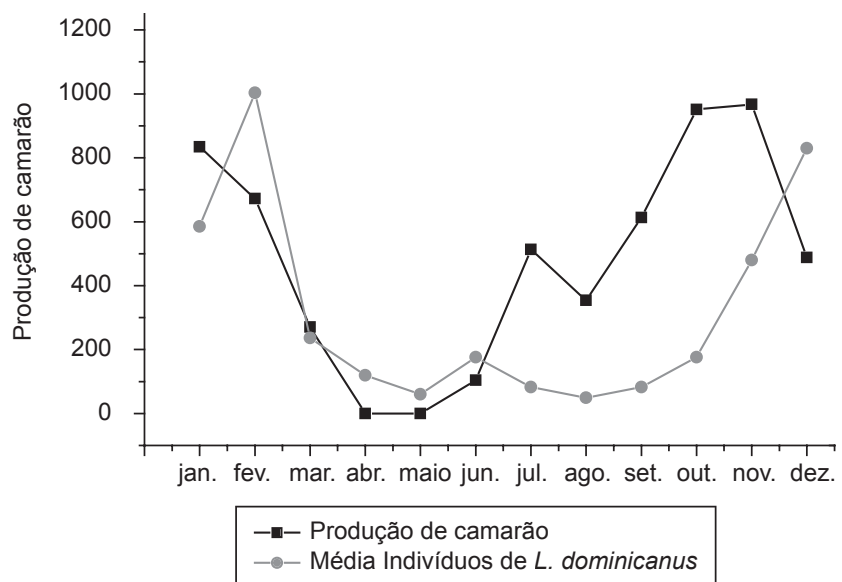

Figura 10. Variação da produção do camarão-sete-barbas (Xiphopenaeus kroyeri) e das médias de indivíduos de L. dominicanus, nas cinco áreas combinadas do Estuário de Cananéia-Iguape-Ilha Comprida.

Figure 10. Variation of the production of shrimp (Xiphopenaeus kroyeri) and of the individuals averages of $L$. dominicanus, in the five agreed areas of the Cananéia-Iguape-Ilha Comprida estuary.

Foi possível verificar que a ponta sul da Ilha Comprida abriga concentrações de Larus dominicanus muito mais expressivas do que a ponta norte, além de demonstrar a presença da espécie ao longo de todos os 12 meses do ano. Os sítios fixos mais importantes de pouso de Larus dominicanus para o estuário de Cananéia-Iguape-Ilha Comprida são: Arrozal e Boqueirão Sul. Destes o mais expressivo é o Boqueirão Sul, onde as aves permanecem durante os doze meses do ano, em repouso sexual.

A região do estuário de Cananéia-Iguape-Ilha Comprida é importante em termos de produtividade primária. Com base nos dados apresentados e no fato da espécie usar águas costeiras, a hipótese de que é a produtividade do próprio estuário a responsável pelos altos números de $L$. dominicanus, observados durante todo o ano neste estudo, é altamente relevante.

Os aumentos de L. dominicanus nos meses de janeiro, fevereiro e dezembro no estuário podem ser explicados pelo fato dos mesmos estarem interagindo positivamente com a pesca do camarão-sete-barbas (Boqueirão Sul e Baixio do Arrozal). Assim como já registrado para Thalasseus maximus (Barbieri \& Pinna 2007) e para Rincops niger 
(Barbieri 2007), a L. dominicanus aproveita os rejeitos dessa arte de pesca como fonte fácil de alimento. Branco (2001), estudando os descartes da pesca do camarão sete-barbas em Santa Catarina, também registrou a ocorrência de $L$. dominicanus interagindo com os arrasteiros. Furness et al. (1988) e Evans (1984), já discutiram a importância dos descartes da pesca de arrasto como fonte adicional de alimento para aves marinhas, além de considerarem um dos principais fatores que explicam o aumento do número de aves e sua distribuição no Atlântico Norte.

As grandes variações apresentadas nos censos mensais são comuns neste tipo de trabalho, pois as aves fazem intensos movimentos de uma área à outra em busca de locais de alimentação e descanso, que acabam por causar grandes flutuações na abundância. Este fato já foi também documentado por Barrames \& Pereira (1992) e Barbieri \& Mendonça (2005) para Laridae e Scolopacidae.

Os registros de L. dominicanus, formando bandos heteroespecíficos, pode podem estar relacionados com a estratégia de defesa da espécie, pois há várias opções de local de pouso. O hábito de formar bandos com outras espécies já foi observado por Olmos \& Silva (2003) em Cubatão, da mesma forma, Vooren \& Brusque (1999) relatam a presença da espécie na costa do Rio Grande do Sul, em bandos multiespecíficos. Este comportamento parece ser normal de espécies da família Laridae: Thalasseus sandvicensis eurygnathus, Thalasseus maximus, Sterna hirundinacea e Larus dominicanus (Escalante 1970, Novelli 1997). Para este conjunto de uma gaivota e três trinta-réis, toda a extensão das praias da região estuarina lagunar de Cananéia-Iguape-Ilha Comprida têm, durante todo o ano, a função de área de pouso, a partir da qual as aves possuem acesso aos recursos alimentares de toda a região (Barbieri \& Pinna, 2007).

Gaivotas e trinta-réis alimentam-se nas águas estuarinas e costeiras, e pousam nas praias, em bandos, para o descanso e os cuidados da plumagem (Escalante 1970, Vooren \& Chiaradia 1990, Naves 1999, Barbieri \& Mendonça 2008). A disponibilidade de praias adequadas para o pouso é condição fundamental para a presença destas aves em determinada região (Vooren \& Brusque 1999, Barbieri \& Mendonça 2005, Barbieri \& Pinna 2005). Portanto, essas áreas de pouso deveriam ser mapeadas, para fornecer subsídios a políticas de conservação, bem como ter visitação e uso restrito.

\section{Agradecimentos}

Agradecemos aos técnicos do Instituto de Pesca da Secretaria da Agricultura e Abastecimento do Estado de São Paulo, Núcleo de Pesquisa do Litoral Sul.: Antônio Domingues Pires, Antônio Carlos de Almeida, Eduardo Antônio Hoff, Onésio Veríssimo e Sergio Cunha Xavier, pela ajuda e coleta de dados, em diversas etapas do trabalho.

\section{Referências Bibliográficas}

BARAMES, G. \& PEREIRA, A. 1992. Abundancia y fluctuaciones de aves limicolas (Charadriiformes) en una playa fangosa de Chomes, Costa Rica. Rev. Biol. Trop. 40(2): 303-307.

BARBIERI, E., MENDONÇA, J.T. \& XAVIER, S.C. 2003. Importance of Ilha Comprida (São Paulo State, Brazil) for the Sanderlings (Calidris alba) migration. J. Coast. Res. (Spec. Issue) 35(1): 65-68.

BARBIERI, E. \& MENDONÇA, J.T. 2005. Distribuition and abundance of Charadridae at Ilha Comprida, São Paulo State, Brazil. J. Coast. Res. 21(2): 1-10

BARBIERI, E. \& PINNA, F.V. 2005. Distribuição da Batuíra-de-coleira (Charadrius collaris) durante o período de 1999 a 2001 na praia da Ilha Comprida. Rev. Bras. Ornitol. 13(2): 25-31.

BARBIERI, E. \& MENDONÇA, J. T. 2008. Seasonal abundance and distribution of Larids at Ilha Comprida (São Paulo State, Brazil). J. Coast. Res. 24 (1): 70-78

BARBIERI, E. \& PINNA, F. V. 2007. Distribuição do Trinta-réis-real (Thalasseus maximus) durante 2005 no estuário de Cananéia-Iguape-Ilha Comprida. Ornitol. Neotrop. 18 (1): 99-110.
BARBIERI, E. 2007. Variação sazonal e abundância de Rynchops niger no estuário de Cananéia-Iguape-Ilha Comprida, São Paulo. Biota Neotropica 7 (2): http://www.biotaneotropica.org.br/v7n2/pt/ abstract?article + bn00207022007

BRANCO, J. O. 2001. Descarte da pesca do camarão sete-barbas como fonte de alimento para aves marinhas. Rev. Bras. Zool. 18(2): 293-300.

BRANCO, J.O. \& EBERT, L.A. 2002. Estrutura populacional de Larus dominicanus Lichtenstein, 1823 no estuário do Saco da Fazenda, Itajaí, SC. Ararajuba 10(1): 79-82.

BURGER, J. \& GOCHFELD, M. 1996. Family Laridae (Gulls) p. 572-623. In Handbook of the birds of the world vol.3. (J. del Hoyo, A. Elliott \& J. Sargatal Eds.). Lynx Ediciones, Barcelona.

BIBBY, J.C., BURGUES, N.D. \& HILL, D.A. 1992. Bird census techniques. Academic Press, London, UK. p. 257.

CRAWFORD, R.J.M., COOPER, J., SHULDON, E. P. A. 1982. Distribuition, population size, breeding and conservation of on estuary. Ornis Scand 16(1): 245-252.

ESCALANTE, R. 1970. Aves marinhas del Rio de La Plata y aguas vecinas del oceano Atlantico. Barreiro e Ramos, Montevideo, Uruguai.

EVANS, P.G.H. 1984. Status and conservation of seabirds in northwest Europe (excluding Norway and USSR). p. 29-321. In Status and conservation of world's seabirds. International Council for Bird Preservation, (Croxall, J.P., Evans, P.G.H. \& Scheiber, R.W. eds.).Cambridge, UK.

FAVERO, M., SILVA, P., FERREYRA, G. 1997. Trophic relationship between the Kelp gull and the Antartic limpet at King George Island (South Shetland Island, Antarctica) during the breeding season. Polar Biol. 17(2): 431-436.

FOWLER, J. \& COHEN, J. 1988. Statistics for ornithologists. British Trust for Ornithology. Tring, UK.

FURNESS, R.W., HUDSON, A.V., ENSOR, E. 1988. Interactions between scavenging seabirds and commercial fisheries around the British Isles. p. 240-268. In Seabirds \& other marine vertebrates: competition, predation and other interactions, (Burger, J. Ed.). Columbia Univ. Press, New York, New York.

GIACCARDI, M., YORIO, P., LIZURUME, E. 1997. Patrones estacionales de la gaivota cocinera (Larus dominicanus) em um basural Patagónico y sus relaciones com el manejo de residuos urbanos y pesqueros. Ornitologia Neotropical. 8(2): 77-84.

HOCKEY, P.A.R., RYAN, R.G., BOSMAN, A.L 1989. Age-related intraspecific kleptoparasitism and foraging sucess of kelp gulls Larus dominicanus. Ardea 77(2): 205-210.

MOREIRA, F. 1995. Diet of Black-headed Gulls Larus ridibundus on emerged intertidal areas in the Tagus estuary (Portugal): predation or grazing? Journal of Avian Biology 26(2): 277-282

NOVELLI, R. 1997. Aves marinhas costeiras do Brasil (Identificação e Biologia). Cinco Continente Editora Ltda, Porto Alegre, RS, Brasil. p. 91.

OLMOS, F. \& SILVA e SILVA, R. 2003. Guará: ambiente, flora e fauna dos manguezais de Santos-Cubatão. Empresa das artes, São Paulo, Brasil.

ROSÁRIO, L.A. 2004. Um outro olhar da via expressa sul. Edição da autora, Florianópolis, Brasil.

SICK, H. 1997. Introdução a Ornitologia Brasileira. Editora Gunabara, Rio de Janeiro, RJ, Brasil. p. 843.

WATSON, G.E. 1975. Birds of the Antarctic and Subantarctic. American Geophysical Union. Washington, DC.

VOOREN, C. \& CHIARADIA, A. 1990. Seasonal abundance and behaviour of costal birds on Cassino Beach, Brazil. Ornitologia Neotropical, Québec, 1(1): 9-24.

VOOREN, C.M. \& BRUSQUE, L.F. 1999. As aves do ambiente costeiro do Brasil: Biodiversidade e conservação. http: www.bdt.org.br/workshop/ costa/plat_continental (acesso em 12/11/2006). 